Pacific Journal of Mathematics

A NOTE ON MEISTERS AND OLECH'S PROOF OF THE
GLOBAL ASYMPTOTIC STABILITY JACOBIAN CONJECTURE 


\title{
A NOTE ON MEISTERS AND OLECH'S PROOF OF THE GLOBAL ASYMPTOTIC STABILITY JACOBIAN CONJECTURE
}

\author{
ARNO VAN DEN ESSEN
}

\begin{abstract}
Let $f: \mathbb{R}^{n} \rightarrow \mathbb{R}^{n}$ be a $C^{1}$-vector field with $f(0)=0$. For $p \in \mathbb{R}^{n}$ let $J f(p)$ denote its Jacobian matrix evaluated at $p$. Then it is a well-known result, due to Lyapunov, that the origin is a locally asymptotic rest point of the non-linear autonomous system of ordinary differential equations $\dot{x}=f(x)$ if the origin is a locally asymptotic rest point of the linearized system $\dot{y}=J f(0) y$ (or equivalently if all eigenvalues of the matrix $J f(0)$ have negative real parts).

In 1960 it was conjectured by Markus and Yamabe that the origin is a globally asymptotic rest point $\dot{x}=f(x)$ if for each $p \in \mathbb{R}^{n}$ the orgin is a locally asymptotic rest point of the linearized system $\dot{y}=J f(p) y$. Until now this conjecture is still open. However in 1988 Meisters and Olech proved this conjecture for two-dimensional polynomial vector fields $f: \mathbb{R}^{2} \rightarrow \mathbb{R}^{2}$. The proof is an immediate consequence of earlier results of Olech, (1963) and the proposition below. The main result of this paper (Theorem 1) generalizes the proposition to polynomial maps $F: k^{n} \rightarrow k^{n}$ having the property that $\operatorname{det} J F(x) \neq 0$ for all $x \in k^{n}$ ( $k$ is a field of characteristic zero).
\end{abstract}

Proposition. If $F: \mathbb{R}^{2} \rightarrow \mathbb{R}^{2}$ is a polynomial map such that $\operatorname{det} J F(x) \neq 0$ for all $x \in \mathbb{R}^{2}$, then there exists a positive integer $N$ such that the number of elements in each fiber $F^{-1}(x)\left(x \in \mathbb{R}^{2}\right)$ is bounded by $N$.

The proof of this proposition given by Meisters and Olech uses topological methods. In this note we generalize this result to polynomial maps $F: k^{n} \rightarrow k^{n}$ with the property that $\operatorname{det} J F(x) \neq 0$ for all $x \in k^{n}$ ( $k$ is a field of characteristic zero). Our proof is purely algebraic and uses some well-known techniques from the theory of $\mathscr{D}$-modules. For the reader's convenience we have included a section reviewing some results concerning $\mathscr{D}$-modules.

1. The Main Theorem. Throughout this paper we have the following notations: $k$ is a field of characteristic zero and $F: k^{n} \rightarrow k^{n}$ is a polynomial map $(n \geq 1)$ i.e. $F$ is given by coordinate functions $F_{i}$ 
which are elements of the polynomial ring $k[X]:=k\left[X_{1}, \ldots, X_{n}\right]$. The determinant of the Jacobian matrix $J F:=\left(\partial F_{i} / \partial X_{j}\right)$ we denote by $\Delta$. So $\Delta \in k[X]$. For $a \in k[X], \operatorname{deg} a$ denotes the (total) degree of $a$. Finally $\operatorname{deg} F:=\max \operatorname{deg} F_{i}$. Now we can formulate the main result of this note:

THeOREM 1. If $F: k^{n} \rightarrow k^{n}$ is a polynomial map with the property that det $J F(x) \neq 0$ for all $x \in k^{n}$, then there exists a positive integer $N$ such that for each $x \in k^{n}$ the number of elements in the fiber $F^{-1}(x)$ is bounded by $N$.

The proof of this theorem uses some well-known techniques from the theory of $\mathscr{D}$-modules (due to I. N. Bernstein, [1]). A review of some of the results concerning $A_{n}$-modules is given in $\S 2$.

1.1. The $A_{n}$-module structure on $k[X]\left[\Delta^{-1}\right]$. From now on $F=$ $\left(F_{1}, \ldots, F_{n}\right)$ is a polynomial map from $k^{n}$ to $k^{n}$ such that $\Delta(x) \neq$ 0 for all $x \in k^{n}$. In particular we have $\Delta \neq 0$ so the elements $F_{1}, \ldots, F_{n}$ are algebraically independent over $k$ by [6], satz 61. So $k[F]:=k\left[F_{1}, \ldots, F_{n}\right]$ is a subring of $k[X]$ isomorphic to $k[X]$. First we define derivations on the localization $k[X]\left[\Delta^{-1}\right]$, denoted by $\partial / \partial F_{i}$, which satisfy

$$
\frac{\partial}{\partial F_{i}}\left(F_{j}\right)=\delta_{i j}, \quad \text { all } 1 \leq i, j \leq n .
$$

Therefore set $\partial / \partial F_{i}=\sum_{k} a_{i k}\left(\partial / \partial X_{k}\right)$, and we try to find elements $a_{i k} \in k[X]\left[\Delta^{-1}\right]$ such that (1.2) is satisfied. In matrix notation (1.2) is equivalent to

$$
\left(a_{i k}\right)(J F)^{T}=I_{n} .
$$

Since $\operatorname{det}(J F)^{T}=\operatorname{det} J F=\Delta \neq 0$ we can solve the $a_{i k}$ uniquely in $k[X]\left[\Delta^{-1}\right]$. In fact by Cramer's rule we find

$$
\text { (1.4) } \Delta a_{i k} \in k[X] \quad \text { and } \operatorname{deg} \Delta a_{i k} \leq(n-1) \operatorname{deg} F, \quad \text { all } i, k \text {. }
$$

Now we claim that the $k$-derivations $\partial / \partial F_{i}$ commute pairwise on $k[X]\left[\Delta^{-1}\right]$. Therefore let $\tau:=\left[\partial / \partial F_{i}, \partial / \partial F_{j}\right]$ be the commutator of $\partial / \partial F_{i}$ and $\partial / \partial F_{j}$. Then $\tau$ is a $k$-derivation on $k[X]\left[\Delta^{-1}\right]$ and it is zero on $k[F]$ (since $\tau\left(F_{p}\right)=0$ for all $p$ ). Consequently, the unique extension of $\tau$ to the completion $k[[F]]$ is also zero. However by the local inversion theorem ([7], $\S 4$, no. 5. Proposition 5) $k[[F]]=k[[X]]$ (for this last statement we assumed that $F(0)=0$, which is a harmless assumption since $\partial / \partial F_{i}=\partial / \partial\left(F_{i}+\lambda\right)$ for all $\left.\lambda \in k\right)$. So $\tau$ is 
zero on $k[[X]]$ and hence on the subring $k[X]\left[\Delta^{-1}\right](\Delta(0) \neq 0$, so $\left.\Delta^{-1} \in k[[X]]\right)$, which proves the claim.

The results above enable us to endow $k[X]\left[\Delta^{-1}\right]$ with a left $A_{n}=$ $k\left[Y_{1}, \ldots, Y_{n}, \partial_{1}, \ldots, \partial_{n}\right]$-module structure, as follows: Define $Y_{i} \cdot g:=F_{i} g, \quad \partial_{i} \cdot g=\frac{\partial g}{\partial F_{i}} \quad$ for all $1 \leq i \leq n, \quad$ all $g \in k[X]\left[\Delta^{-1}\right]$.

The left $A_{n}$-module associated to $F$ in this way we denote by $M(F)$.

LEMMA 1.5. $M(F)$ possesses an $(n, e(F))$-filtration, where $e(F)=$ $2^{n}(2 n \operatorname{deg} F+1)^{n}$.

Proof. Put $d:=\operatorname{deg} F$. For each $v \in \mathbb{Z}, v \geq 0$ we define

$$
\Gamma_{v}:=\left\{q \Delta^{-2 v} \in k[X]\left[\Delta^{-1}\right] \mid \operatorname{deg} q \leq 2 v(2 n d+1)\right\} .
$$

By definition $\operatorname{dim}_{k} \Gamma_{v}$ is the dimension of the $k$-vector space of all polynomials in $k[X]$ of degree $\leq 2 v(2 n d+1)$, which implies

$$
\operatorname{dim}_{k} \Gamma_{v} \leq \frac{2^{n}(2 n d+1)^{n}}{n !} v^{n}+\mathscr{O}\left(v^{n-1}\right) \text {. }
$$

So it suffices to prove that $\left\{\Gamma_{n}\right\}$ is a filtration on $M(F)$. We first show that $\partial_{i} \Gamma_{v} \subset \Gamma_{v+1}$ (the inclusion $x_{i} \Gamma_{v} \subset \Gamma_{v+1}$ is proved in a similar way). So let $g=q \Delta^{-2 v} \in \Gamma_{v}$. Then

$$
\partial_{i} g=\frac{\partial q}{\partial F_{i}} \Delta^{-2 v}+q(-2 v) \Delta^{-2 v-1} \frac{\partial \Delta}{\partial F_{i}} \text {. }
$$

By (1.4) we know

$$
\frac{\partial}{\partial F_{i}}=\frac{1}{\Delta} \sum_{k} \Delta a_{i k} \frac{\partial}{\partial X_{k}} \quad \text { and }
$$

$\Delta a_{i k} \in k[X] \quad$ with $\operatorname{deg} \Delta a_{i k} \leq(n-1) d$.

So

$$
\partial_{i} g=\left(\Delta \sum_{k} \Delta a_{i k} \frac{\partial q}{\partial X_{k}}+(-2 v) q \sum_{k} \Delta a_{i k} \frac{\partial \Delta}{\partial X_{k}}\right) \Delta^{-2(v+1)}
$$

Using $\operatorname{deg} \Delta \leq n d$ and $\operatorname{deg} \Delta a_{i k} \leq(n-1) d$ we conclude that $\partial_{i} g \in$ $\Gamma_{v+1}$. Finally we show that $U \Gamma_{v}=M(F)$. So let $q \Delta^{-r} \in k[X]\left[\Delta^{-1}\right]$ with $\operatorname{deg} q=s$ and $r \geq 0$. Let $v \geq \max (r, s)$. Then

$$
q \Delta^{-r}=q\left(\Delta^{2 v-r}\right) \Delta^{-2 v} \quad \text { and }
$$

$\operatorname{deg} q \Delta^{2 v-r} \leq s+(2 v-r) n d \leq s+2 v n d \leq 2 v(2 n d+1)$

since $v \geq s$. So $q \Delta^{-r} \in \Gamma_{v}$, which completes the proof. 
Proof of Theorem 1. (i) Let $x \in k^{n}$. Then the number of elements in the fiber $F^{-1}(x)$ is equal to the number of zeros of the ideal $\left(F_{1}\right.$ $\left.x_{1}, \ldots, F_{n}-x_{n}\right)$. Therefore we consider the polynomial map $F-x$ and form its left $A_{n}$-module $M(x):=M(F-x)$. (Observe that $\operatorname{det} J(F-x)=\operatorname{det} J F=\Delta$ has no zeros in $k^{n}$.) By Lemma 1.5 $M(x)$ possesses an $(n, e(x))$-filtration, where

$$
e(x)=2^{n}(2 n \operatorname{deg}(F-x)+1)^{n}=2^{n}(2 n \operatorname{deg} F+1)^{n} .
$$

So by Corollary $2.4 M(x) / \sum_{i}\left(F_{i}-x_{i}\right) M(x)$ is a finite dimensional $k$-vector space with dimension bounded by $N_{0}:=2^{n}(2 n \operatorname{deg} F+1)^{n}$, which is independent of $x$ ! So

$$
\operatorname{dim}_{k} k[X]\left[\Delta^{-1}\right] / \sum_{i}\left(F_{i}-x_{i}\right) k[X]\left[\Delta^{-1}\right] \leq N_{0} \quad \text { for all } x \in k^{n} .
$$

Consequently the residue classes of $1, X_{1}, X_{1}^{2}, \ldots, X_{1}^{N_{0}}$ must be linearly dependent over $k$. So there exists a non-zero polynomial $g\left(X_{1}\right)$ $\in k\left[X_{1}\right]$ of degree $\leq N_{0}$ and a positive integer $\rho$ such that $\Delta^{\rho} g\left(X_{1}\right)$ $\in \sum k[X]\left(F_{i}-x_{i}\right)$.

(ii) Now let $p=\left(p_{1}, \ldots, p_{n}\right) \in k^{n}$ such that $F(p)=x$; i.e. $F_{i}(p)=x_{i}$ for all $i$. Then $\Delta(p)^{\rho} g\left(p_{1}\right)=0$. Since $\Delta$ has no zeros on $k^{n}$ it follows that $g\left(p_{1}\right)=0$. So there are at most $N_{0}$ possibilities for the first coordinate of $p$ (since $\operatorname{deg} g \leq N_{0}$ ). Arguing in a similar way for the other coordinates of $p$ we conclude that the number of $p \in k^{n}$ with $F(p)=x$ is bounded by $N:=N_{0}^{n}$.

Comment. It was kindly pointed out to me by Professor J. Bochnak that for some special fields $k$ such as $\mathbb{R}, \mathbb{C}$, real closed or algebraically closed fields, Theorem 1 is a consequence of the following result.

TheOREM 1.6. Let $F: \mathbb{R}^{n} \rightarrow \mathbb{R}^{p}$ be a polynomial map of degree $d$ such that $F^{-1}(x)$ is finite for each $x \in \mathbb{R}^{p}$. Then the number of elements in each fiber $F^{-1}(x)$ is bounded by $d(2 d-1)^{n-1}$.

This theorem is a very special case of Theorem 11.5 .2 (p. 243) of [8]. To see that Theorem 1.6 implies Theorem 1 one only needs to observe that the condition det $J F(x) \neq 0$ for all $x \in \mathbb{R}^{n}$ implies that each fiber $F^{-1}(x)$ is discrete (by the implicit function theorem) and that obviously $F^{-1}(x)$ is an algebraic subset of $\mathbb{R}^{n}$ and hence has a finite number of connected components. So $F^{-1}(x)$ is finite.

2. A review of some results concerning $A_{n}$-modules. All results of this section come from I. N. Bernstein's work in [1] and can also be found in Chapter I of [2]. 
Let $A_{n}:=k\left[Y_{1}, \ldots, Y_{n}, \partial_{1}, \ldots, \partial_{n}\right]$ be the $n$th Weyl-algebra, i.e. the $k$-algebra with relations $\left[Y_{i}, Y_{j}\right]=\left[\partial_{i}, \partial_{j}\right]=0$ and $\left[\partial_{i}, Y_{j}\right]=\delta_{i j}$ for all $1 \leq i, j \leq n$. It is a filtered ring with filtration $\left\{T_{v}\right\}_{v=0}^{\infty}$ where $T_{v}$ is the $k$-vector space generated by the monomials $Y^{\alpha} \partial^{\beta}$ with $|\alpha|+|\beta| \leq v$ (with the usual multi-index notation). Let $M$ be a left $A_{n}$-module. A filtration $\Gamma$ on $M$ is an increasing sequence $\Gamma_{0} \subset \Gamma_{1} \subset \Gamma_{2} \subset \cdots$ of finite dimensional $k$-subspaces of $M$ such that $\bigcup T_{v}=M$ and $T_{k} \Gamma_{v} \subset \Gamma_{v+k}$ for all $k, v \geq 0$. Such a filtration is called good if there exist $m_{1}, \ldots, m_{s} \in M$ and $n_{1}, \ldots, n_{s} \in \mathbb{Z}$ such that $\Gamma_{v}=\sum T_{v-n_{i}} m_{i}$ for all $v \geq 0$ (by definition $T_{-v}=0$ for all $v \geq 1$ ). One readily verifies that an $A_{n}$-module possesses a good filtration if and only if it is finitely generated. Furthermore we have

Proposition 2.1 ([2], Corollary 3.3, Chapter I). If $\Gamma$ is a good filtration on a finitely generated left $A_{n}$-module $M$, then there exist an integer $d \geq 0$ and rational numbers $a_{0}, \ldots, a_{d}$ such that

$$
\operatorname{dim}_{k} \Gamma_{v}=a_{d} v^{d}+a_{d-1} v^{d-1}+\cdots+a_{0}, \quad \text { for all large } v .
$$

Furthermore $d ! a_{d}$ is an integer $\geq 1$.

The crucial point is that the integers $d$ and $d ! a_{d}$ are independent of the choice of the good filtration; they form two important invariants of the $A_{n}$-module $M$, called the dimension and the multiplicity of $M$, denoted $d(M)$, resp. $e(M)$. The fundamental Bernstein inequality asserts that $d(M) \geq n$ for every non-zero $A_{n}$-module $M$ of finite type! The non-zero $A_{n}$-modules of finite type having the minimal dimension $n$ are called holonomic $A_{n}$-modules. They play a very important role in the theory of $\mathscr{D}$-modules. A useful fact is that a holonomic $A_{n}$-module with multiplicity $e(M)$ has a finite length, bounded by $e(M)$.

To decide if a given $A_{n}$-module is holonomic, there exists a very powerful criterion. Before we describe it we introduce some terminology. Let $M$ be a left $A_{n}$-module, not necessary of finite type. A filtration $\Gamma$ on $M$ is called a $(d, e)$-filtration if $\operatorname{dim}_{k} \Gamma_{n} \leq \frac{e}{d !} v^{d}+\mathscr{O}\left(v^{d-1}\right)$ where $d \geq 0$ and $e \geq 1$ are integers. Observe that if $M$ is holonomic it possesses an $(n, e)$-filtration (namely take any good filtration on $M$ and apply Proposition 2.1). However the converse also holds i.e.

TheOREM 2.2 ([2], Theorem 5.4, Chapter I). Let $M$ be an arbitrary $A_{n}$-module (so we don't assume $M$ to be of finite type). If $M$ possesses 
an $(n, e)$-filtration for some integer $e \geq 1$, then $M$ is holonomic (and hence of finite type). Furthermore $e(M) \leq e$.

Now consider the multiplication $Y_{n}: M \rightarrow M$. Then

$$
\text { coker } Y_{n}:=M / Y_{n} M
$$

can be given the structure of a left $A_{n-1}=k\left[Y_{1}, \ldots, Y_{n-1} \partial_{1}, \ldots, \partial_{n-1}\right]-$ module by putting $\partial_{i}\left(m+Y_{n} M\right):=\partial_{i} m+Y_{n} M$. If $n=1$ we put $A_{0}:=k$.

THEOREM 2.3 ([2], Theorem 6.2, Chapter I). Let $M$ be an $A_{n}$ module with an $(n, e)$-filtration. Then $M / Y_{n} M$ is an $A_{n-1}$-module with an $(n-1, e)$-filtration. If $n=1$ it means that $M / Y_{n} M$ is a $k$-vector space of dimension $\leq e$.

\section{By applying this result $n$-times we arrive at}

COROllary 2.4. Let $M$ be an $A_{n}$-module with an $(n, e)$-filtration. Then $M / \sum_{i} Y_{i} M$ is a finite dimensional $k$-vector space with dimension bounded by $e$.

\section{REFERENCES}

[1] I. N. Bernstein, The analytic continuation of generalized functions with respect to a parameter, Funktsional. Anal. i Prilozhen., 6 (1972), 26-40. English transl. in Functional Anal. Appl., 6 (1972), 273-285.

[2] J.-E. Björk, Rings of Differential Operators, Vol. 21, North-Holland Mathematical Library (1979).

[3] L. Markus and H. Yamabe, Global stability criteria for differential systems, Osaka Math. J., 12 (2) (1960), 305-317.

[4] G. Meisters and C. Olech, Solution of the Global Asymptotic Stability Jacobian Conjecture for the Polynomial Case, Analyse Mathématique et Applications, Gauthier-Villars, Paris (1988), 373-381.

[5] C. Olech, On the global stability of an autonomous system on the plane, Contributions to Differential Equations, 1 (1963), 389-400.

[6] O. Perron, Algebra I, Die Grundlagen, Walter de Gruyter \& Co., Berlin 1951.

[7] N. Bourbaki, Algèbre Commutative, Chapter 3, Hermann Paris.

[8] J. Bochnak, M. Coste and M. -F. Roy, Géometrie algébrique réelle, Ergebnisse der Math. u.i. Grenzgebieten, Springer-Verlag, Berlin-Heidelberg-New York, 1987.

Received April 16, 1990 and in revised form September 21, 1990.

KATHOLIEKE UNIVERSITEIT

TOERNOOIVELD

NiJMegen 6525 ED, The Netherlands 


\section{PACIFIC JOURNAL OF MATHEMATICS EDITORS}

\author{
V. S. VARADARAJAN \\ (Managing Editor) \\ University of California \\ Los Angeles, CA 90024-1555-05 \\ Herbert Clemens \\ University of Utah \\ Salt Lake City, UT 84112 \\ THOMAS ENRIGHT \\ University of California, San Diego \\ La Jolla, CA 92093
}

Nicholas ERcolani

University of Arizona

Tucson, AZ 85721

R. FINN

Stanford University

Stanford, CA 94305

VAUGHAN F. R. JONES

University of California

Berkeley, CA 94720

STEVEN KeRCKHOFF

Stanford University

Stanford, CA 94305

\section{C. MOORE \\ University of California \\ Berkeley, CA 94720}

MARTIN SChaRLEMANN

University of California

Santa Barbara, CA 93106

HAROLd STARK

University of California, San Diego

La Jolla, CA 92093

\section{ASSOCIATE EDITORS}

\begin{tabular}{|c|c|c|c|c|}
\hline ARENS & $\begin{array}{l}\text { E. F. BECKENBACH } \\
(1906-1982)\end{array}$ & NeumanN & $\begin{array}{l}\text { F. Wolf } \\
(1904-1989)\end{array}$ & K. Yoshida \\
\hline \multicolumn{5}{|c|}{ SUPPORTING INSTITUTIONS } \\
\hline \multirow{2}{*}{\multicolumn{2}{|c|}{$\begin{array}{l}\text { IVERSITY OF ARIZONA } \\
\text { IIVERSITY OF BRITISH COLUMBIA }\end{array}$}} & UNIVERS & Y OF OREGON & \\
\hline & & UNIVERS & OF SOUTHER & IIA \\
\hline \multicolumn{2}{|c|}{ LIFORNIA INSTITUTE OF TECHNOLOGY } & STANFO & UNIVER & \\
\hline \multirow{2}{*}{\multicolumn{2}{|c|}{$\begin{array}{l}\text { IVERSITY OF CALIFORNIA } \\
\text { ONTANA STATE UNIVERSITY }\end{array}$}} & UNIVERS & OF HAWAII & \\
\hline \multirow{2}{*}{\multicolumn{2}{|c|}{$\begin{array}{l}\text { ONTANA STATE UNIVERSITY } \\
\text { IIVERSITY OF NEVADA, RENO }\end{array}$}} & UNIVERS & YF TOKYO & \\
\hline & & UNIVERS & I OF UTAH & \\
\hline \multirow{2}{*}{\multicolumn{2}{|c|}{$\begin{array}{l}\text { W MEXICO STATE UNIVERSITY } \\
\text { EGON STATE UNIVERSITY }\end{array}$}} & WASHING & N STATE UNI & SITY \\
\hline & & UNIVERS & NASI & \\
\hline
\end{tabular}

The Supporting Institutions listed above contribute to the cost of publication of this Journal, but they are not owners or publishers and have no responsibility for its content or policies.

Mathematical papers intended for publication in the Pacific Journal of Mathematics should be in typed form or offset-reproduced (not dittoed), double spaced with large margins. Please do not use built up fractions in the text of the manuscript. However, you may use them in the displayed equations. Underline Greek letters in red, German in green, and script in blue. The first paragraph must be capable of being used separately as a synopsis of the entire paper. In particular it should contain no bibliographic references. Please propose a heading for the odd numbered pages of less than 35 characters. Manuscripts, in triplicate, may be sent to any one of the editors. Please classify according to the 1991 Mathematics Subject Classification scheme which can be found in the December index volumes of Mathematical Reviews. Supply name and address of author to whom proofs should be sent. All other communications should be addressed to the managing editor, or Elaine Barth, University of California, Los Angeles, California 90024-1555-05.

There are page-charges associated with articles appearing in the Pacific Journal of Mathematics. These charges are expected to be paid by the author's University, Government Agency or Company. If the author or authors do not have access to such Institutional support these charges are waived. Single authors will receive 50 free reprints; joint authors will receive a total of 100 free reprints. Additional copies may be obtained at cost in multiples of 50 .

The Pacific Journal of Mathematics (ISSN 0030-8730) is published monthly except for July and August. Regular subscription rate: $\$ 190.00$ a year (10 issues). Special rate: $\$ 95.00$ a year to individual members of supporting institutions.

Subscriptions, orders for numbers issued in the last three calendar years, and changes of address should be sent to Pacific Journal of Mathematics, P.O. Box 969, Carmel Valley, CA 93924, U.S.A. Old back numbers obtainable from Kraus Periodicals Co., Route 100, Millwood, NY 10546.

The Pacific Journal of Mathematics at P.O. Box 969, Carmel Valley, CA 93924 (ISSN 0030-8730) is published monthly except for July and August. Second-class postage paid at Carmel Valley, Californiä 93924, and additional mailing offices. Postmaster: send address changes to Pacific Journal of Mathematics, P.O. Box 969, Carmel Valley, CA 93924.

\section{PUBLISHED BY PACIFIC JOURNAL OF MATHEMATICS, A NON-PROFIT CORPORATION}




\section{Pacific Journal of Mathematics}

\section{Vol. 151, No. $2 \quad$ December, 1991}

Michael G. Eastwood and A. M. Pilato, On the density of twistor

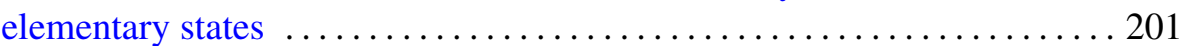

Brian E. Forrest, Arens regularity and discrete groups $\ldots \ldots \ldots \ldots \ldots \ldots 217$

Yu Li Fu, On Lipschitz stability for F.D.E ..................... 229

Douglas Austin Hensley, The largest digit in the continued fraction expansion of a rational number $\ldots \ldots \ldots \ldots \ldots \ldots \ldots \ldots \ldots \ldots \ldots . \ldots 237$

Uwe Kaiser, Link homotopy in $\mathbb{R}^{3}$ and $S^{3}$

Ronald Leslie Lipsman, The Penney-Fujiwara Plancherel formula for abelian symmetric spaces and completely solvable homogeneous

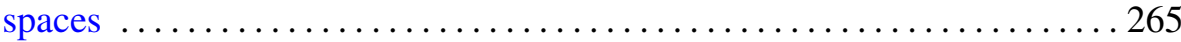

Florin G. Radulescu, Singularity of the radial subalgebra of $\mathscr{L}\left(F_{N}\right)$ and the

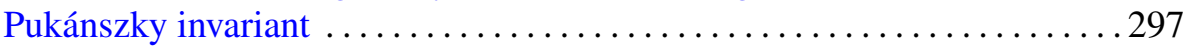

Albert Jeu-Liang Sheu, The structure of twisted SU(3) groups $\ldots . \ldots \ldots . \ldots 307$

Morwen Thistlethwaite, On the algebraic part of an alternating link . . . . . 317

Thomas (Toma) V. Tonev, Multi-tuple hulls .................. 335

Arno van den Essen, A note on Meisters and Olech's proof of the global asymptotic stability Jacobian conjecture ..................... 351

Hendrik J. van Maldeghem, A characterization of the finite Moufang hexagons by generalized homologies

Bun Wong, A note on homotopy complex surfaces with negative tangent bundles

Chung-Tao Yang, Any Blaschke manifold of the homotopy type of $\mathbf{C} P^{n}$ has the right volume 